\title{
Incidence of Radiologically Isolated Syndrome: A Population-Based Study
}

(D). Forslin, (D). Granberg, (D) A. Antwan Jumah, (D). Shams, (DP. Aspelin, (D). Kristoffersen-Wiberg, (D). Martola, and (D). Fredrikson

\begin{abstract}
BACKGROUND AND PURPOSE: Incidental MR imaging findings resembling MS in asymptomatic individuals, fulfilling the Okuda criteria, are termed "radiologically isolated syndrome." Those with radiologically isolated syndrome are at high risk of their condition converting to MS. The epidemiology of radiologically isolated syndrome remains largely unknown, and there are no population-based studies, to our knowledge. Our aim was to study the population-based incidence of radiologically isolated syndrome in a high-incidence region for MS and to evaluate the effect on radiologically isolated syndrome incidence when revising the original radiologically isolated syndrome criteria by using the latest radiologic classification for dissemination in space.
\end{abstract}

MATERIALS AND METHODS: All 2272 brain MR imaging scans in 1907 persons obtained during 2013 in the Swedish county of Västmanland, with a population of 259,000 inhabitants, were blindly evaluated by a senior radiologist and a senior neuroradiologist. The Okuda criteria for radiologically isolated syndrome were applied by using both the Barkhof and Swanton classifications for dissemination in space. Assessments of clinical data were performed by a radiology resident and a senior neurologist.

RESULTS: The cumulative incidence of radiologically isolated syndrome was 2 patients ( $0.1 \%$ ), equaling an incidence rate of 0.8 cases per 100,000 person-years, in a region with an incidence rate of MS of 10.2 cases per 100,000 person-years. There was no difference in the radiologically isolated syndrome incidence rate when applying a modified version of the Okuda criteria by using the newer Swanton classification for dissemination in space.

CONCLUSIONS: Radiologically isolated syndrome is uncommon in a high-incidence region for MS. Adapting the Okuda criteria to use the dissemination in space-Swanton classification may be feasible. Future studies on radiologically isolated syndrome may benefit from a collaborative approach to ensure adequate numbers of participants.

ABBREVIATIONS: DIS = dissemination in space; RIS = radiologically isolated syndrome

D ue to the increased sensitivity, availability, and use of MR imaging, incidental findings have increased during the past decade. $^{1,2}$ Incidental MR imaging findings resembling MS, termed "radiologically isolated syndrome" (RIS), are 1 consequence of the increased use and sensitivity of MR imaging. ${ }^{3}$ RIS was introduced in 2009 by Okuda et $\mathrm{al}^{3}{ }^{3}$ to categorize incidental

Received October 14, 2015; accepted after revision November 20

From the Departments of Clinical Science, Intervention and Technology (Y.F., T.G., S.S., P.A., M.K.-W., J.M.), Radiology (Y.F., T.G., S.S., P.A., M.K.-W., J.M.), Clinical Neuroscience (S.F.), and Neurology (S.F.), Karolinska University Hospital, Stockholm, Sweden; and Department of Radiology (Y.F., T.G., A.A.J.), Västmanland Hospital, Västerås, Sweden.

Contributions: T.G. conceived the study. Y.F., T.G., A.A.J., P.A., M.K.-W., J.M., and S.F. jointly initiated the study. Y.F., T.G., A.A.J., and J.M. designed the study. Y.F., A.A.J., and J.M. performed data acquisition. Y.F., T.G., S.S., M.K.-W., and S.F. analyzed the data. All authors contributed to the interpretation of the data and revised the manuscript. Y.F., T.G., and S.S. drafted the manuscript. Y.F is a guarantor.

This work was funded by the Stockholm County Council and Karolinska Institutet through the regional agreement on medical training and clinical research (ALF grant 20120213).
WM lesions suggestive of demyelinating disease in patients without typical MS symptoms and no better explanation for the MR imaging anomalies. ${ }^{4}$ RIS has, since its emergence, been debated, and the risk of RIS evolving into MS has been investigated. ${ }^{5}$ Nonconventional MR imaging methods and neuropsychological testing have revealed similar findings in RIS and MS. ${ }^{6-10}$ One-third of patients with RIS develop MS in 5 years, implying that RIS, in some cases, constitutes a preclinical stage or subclinical form of MS. These results suggest that the McDonald criteria lack some degree of sensitivity in detecting MS in its earliest phases. This interpretation raises questions about the early pathophysiology of MS and motivates us to investigate to what extent it is possible to

\footnotetext{
Please address correspondence to Yngve Forslin, MD, Department of Clinical Science, Intervention and Technology, Cl-46, Karolinska University Hospital, 14186 Stockholm, Sweden; e-mail: yngve.forslin@ki.se

- Indicates open access to non-subscribers at www.ajnr.org

三 Indicates article with supplemental on-line tables.

http://dx.doi.org/10.3174/ajnr.A4660
} 


$\begin{aligned} & \text { Table 1: Comparison of the } 2 \text { latest radiologic criteria for DIS in } \\
& \text { MS }\end{aligned}$
\begin{tabular}{ll}
\hline DIS Barkhof Classification & \\
\hline At least 3 of & DIS Swanton Classification \\
& 26 \\
$\geq 3$ Periventricular lesion & At least 2 of \\
$\geq 1$ Juxtacortical lesion & $\geq 1$ Periventricular lesion \\
$\geq 1$ Infratentorial or spinal & $\geq 1$ Juxtacortical lesion \\
$\quad$ cord lesion & $\geq 1$ Infratentorial lesion \\
$\geq 1$ Contrast-enhancing or & \\
$\geq 9$ T2 lesions & \\
\hline
\end{tabular}

detect MR imaging findings before the symptomatic onset of MS. It is therefore of further interest to determine the frequency of RIS in clinical practice. ${ }^{3,5,11-18}$

However, despite the interest in RIS, its epidemiology remains largely unclear. Postmortem studies have shown a prevalence of incidental MS findings ranging from $0.06 \%$ to $0.7 \% .^{5,19-21}$ The hospital-based incidence has been estimated between $0.05 \%$ and $0.7 \%{ }^{17,22}$ Our aim was to study the population-based incidence of RIS in a country with a high incidence ( 10.2 per 100,000 person-years) and prevalence $(189 / 100,000)$ of MS. ${ }^{23,24}$ This was done by retrospectively re-evaluating all MR imaging brain examinations performed during 2013 in the Swedish county of Västmanland, with approximately 259,000 inhabitants. ${ }^{25}$ We secondarily aimed to evaluate how implementing the newer Swanton classification for dissemination in space (DIS), from the $2010 \mathrm{Mc}-$ Donald criteria for MS, would affect the incidence of RIS. ${ }^{3,4,26,27}$

\section{MATERIALS AND METHODS \\ Study Population}

Ethics approval was obtained from the regional ethics review board in Stockholm, Sweden. All brain MR images obtained during 2013 in Västmanland County, Sweden, were anonymized and included in the study. Written informed consent was obtained for reviewing the clinical patient charts.

\section{Criteria and Assessments}

The Okuda criteria for RIS, summarized in On-line Table 1, were used. ${ }^{3}$ Similar to a previous study, ${ }^{28}$ radiologic DIS was assessed according to both the original implementation with the Barkhof classification (DIS-Barkhof) ${ }^{4}$ and with the more recent Swanton classification (DIS-Swanton) $)^{26,27}$ to study the effect of the 2 different classifications on the RIS incidence. Table 1 compares the 2 DIS classifications.

The screening of all patients was conducted in several steps, illustrated in Fig 1. First, all brain MR imaging scans obtained in 2013 were anonymized and systematically evaluated by a senior radiologist (A.A.). The assessment was blinded to the original radiologic reading and all clinical information. The primary radiologic screening by A.A. focused strictly on the location, number, and presence of gadolinium enhancement of the WM lesions in regard to the Swanton and Barkhof criteria, respectively, to assess whether the DIS/Okuda A2 criterion was fulfilled and to preserve a high sensitivity for WM anomalies. Thus, no interpretation of the likely cause of the lesions was performed in the initial radiologic screening. Consistent with the study by Liu et $\mathrm{al},{ }^{29} \mathrm{a}$ more liberal definition of juxtacortical lesions "within $3 \mathrm{~mm}$ from the GM-WM border" was used in the initial screening by A.A., to

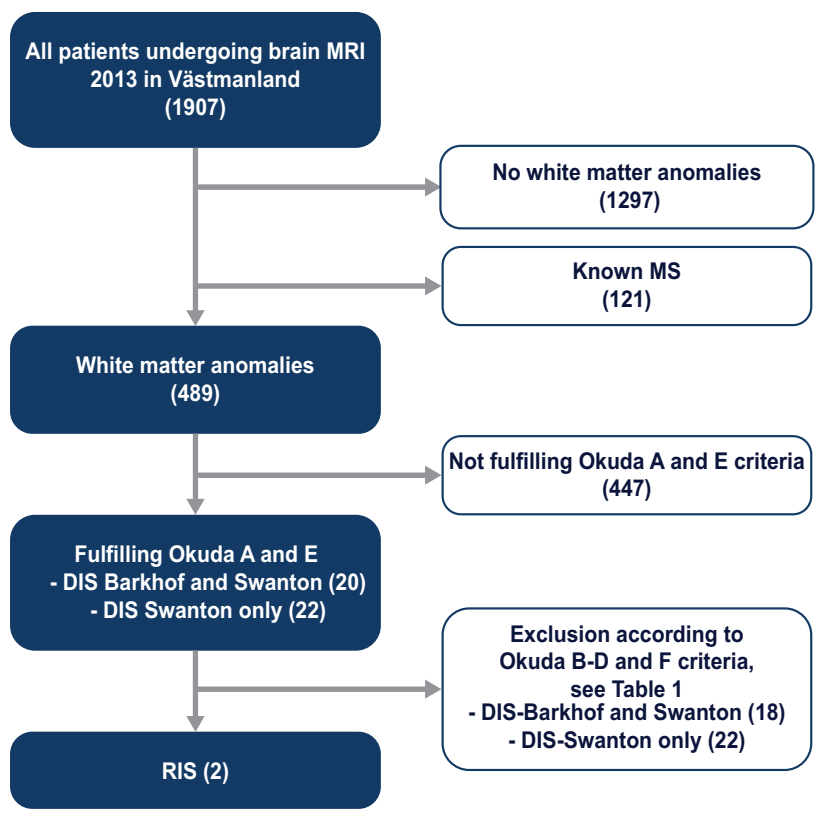

FIG 1. Flow chart of the screening process for identifying patients with RIS.

preserve a high sensitivity for WM anomalies. All patients with DIS were re-evaluated by a senior neuroradiologist (J.M.); for this evaluation, the definition "in direct contact with the cortex" was used when assessing juxtacortical lesions, for higher specificity. J.M. also assessed whether the lesions fulfilled the Okuda A and E criteria.

The clinical information for patients fulfilling the Okuda A and $\mathrm{E}$ criteria was reviewed by a resident in radiology (Y.F.). Exclusions from the study, according to the Okuda B-D and F criteria, were based on the clinical information in the referral notes and, when needed, clinical patient charts. Any uncertain cases and all included patients were discussed with a senior neurologist (S.F.), and final decisions on inclusion/exclusion were made by a consensus of Y.F. and S.F. On-line Table 1 summarizes the causes for exclusion.

\section{MR Imaging Acquisition}

There are 2 MR imaging centers in Västmanland county: the regional hospital in Västerås and Medicinsk Röntgen Eriksborg. Both centers participated in the study. There are $3 \mathrm{MR}$ imaging scanners in Västmanland: one 1.5T Avanto (Siemens, Erlangen, Germany), one 1.5T Symphony (Siemens), and one 3T Ingenia scanner (Philips Healthcare, Best, the Netherlands). All brain MR imaging was performed according to standard clinical protocols based on the clinical query. The MR imaging protocols at the 2 centers and on the 3 scanners varied but always included conventional axial MR imaging sequences (such as T1- and T2-weighted images) with a section thickness of $\leq 5 \mathrm{~mm}$. Naturally, all examinations were individualized for the patients' clinical needs. However, most protocols included FLAIR sequences, and $42 \%$ of the MR images were acquired with the administration of gadolinium-based contrast media. The few protocols that did not include FLAIR were for the following clinical queries: control after intracranial bleeding or aneurysm coiling, arterial angiography, meningioma control, 
Table 2: Overview of MRI scanners, sequences in standard brain protocol, and number of patients receiving gadolinium-based contrast media

\begin{tabular}{lccc}
\hline \multicolumn{1}{c}{ MRI Scanner } & Philips Ingenia & Siemens Symphony & Siemens Avanto \\
\hline Field strength & 3T & 1.5T & 1.5T \\
Standard MRI brain protocol & T1 tra, T1 sag, T2 tra, T2 cor, & T1 tra, T1 sag, T2 tra, T2 cor, & T1 sag, T2 tra, FLAIR cor, \\
& FLAIR tra, DWI tra & FLAIR tra, DWI tra & SWI tra, DWI tra \\
Largest section thickness in any protocol & $5 \mathrm{~mm}$ & $5 \mathrm{~mm}$ & $5 \mathrm{~mm}$ \\
No. of scans with/without contrast media & & $790 / 924$ & $169 / 389$ \\
\hline
\end{tabular}

Note:-Cor indicates coronal; sag, sagittal; tra, transversal.

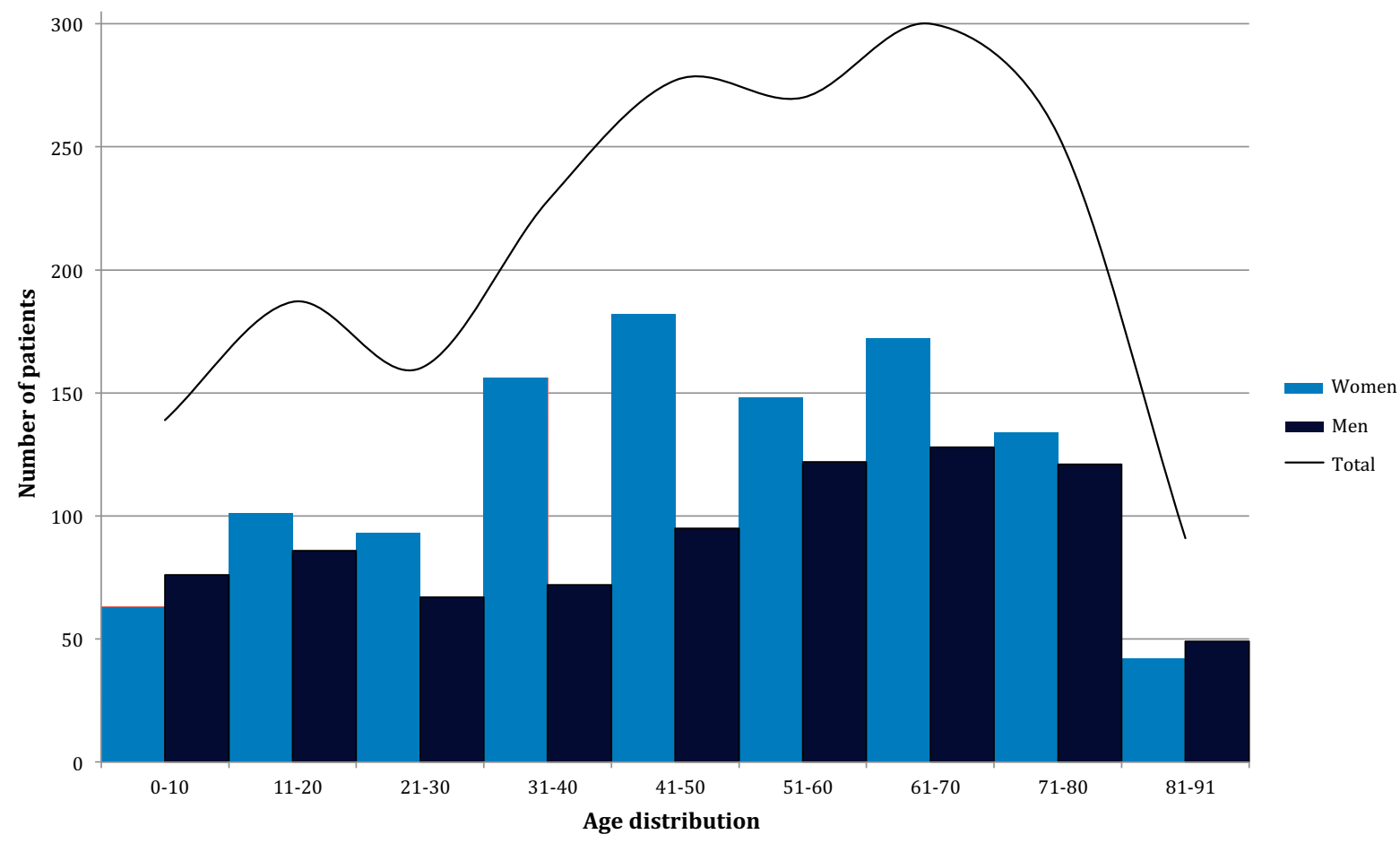

FIG 2. Demographics and distribution of the study population. The ages in the cohort ranged between 0 and 91 years, and the mean age was 47 years (the first and third quartiles were 30 and 66 years, respectively).

and tumor near the pons or a vestibular schwannoma. Table 2 shows the details of the most frequently used standard brain MR imaging protocols, the different MR imaging scanners, and the number of scans with contrast media.

\section{Statistics}

SPSS, Version 22.0 (IBM, Armonk, New York) was used for descriptive statistics, and Excel Mac 2011 14.4.8 (Microsoft, Redmond, Washington), for presenting demographics. The analyses were performed by a resident in radiology (Y.F.).

\section{RESULTS}

\section{Cohort Characteristics}

In 2013, 1907 individuals (1091 females, 816 males) each had between 1 and 6 brain MR imaging scans, with a total of 2272 brain MR imaging scans, equivalent to 877 scans per 100,000 person-years. Figure 2 shows the demography of the study population. An overview of the MR imaging findings is presented in On-line Table 2, which is based on the original radiologic reports (before the study) and, in some cases, complementary information from medical records to specify radiologic findings.

\section{Incidence of RIS}

Of the 1907 patients, 1297 did not have any WM anomalies and 121 were patients with MS with a known diagnosis before the MR imaging. As described in Fig 1, after the exclusion of 447 patients not fulfilling the A and E criteria, 20 patients remained fulfilling both DIS-Barkhof and DIS-Swanton criteria, and 22 patients, fulfilling only the DIS-Swanton criteria. Thus, twice as many patients fulfilled the radiologic criteria for DIS-Swanton $(n=42,2.2 \%)$ in comparison with DIS-Barkhof $(n=20$, $1.0 \%)$.

The main indications for the MR imaging of these patients fulfilling the Okuda A criterion are presented in On-line Table 3, and the causes for exclusion due to clinical reasons (ie, Okuda B-D and F) are presented in On-line Table 1. The most common reason for MR imaging among the 42 patients fulfilling the DIS criteria was headache. Patients with neurologic symptoms consistent with MS were excluded according to the B criterion. Patients with severe dementia were excluded due to the $\mathrm{C}$ criterion. Patients with a history of severe cardiovascular disease or other cardiovascular risk factors, which can lead to cerebral WM anomalies, were excluded according to the Okuda F criteria. CSF analysis had not been performed in most of the excluded patients because 

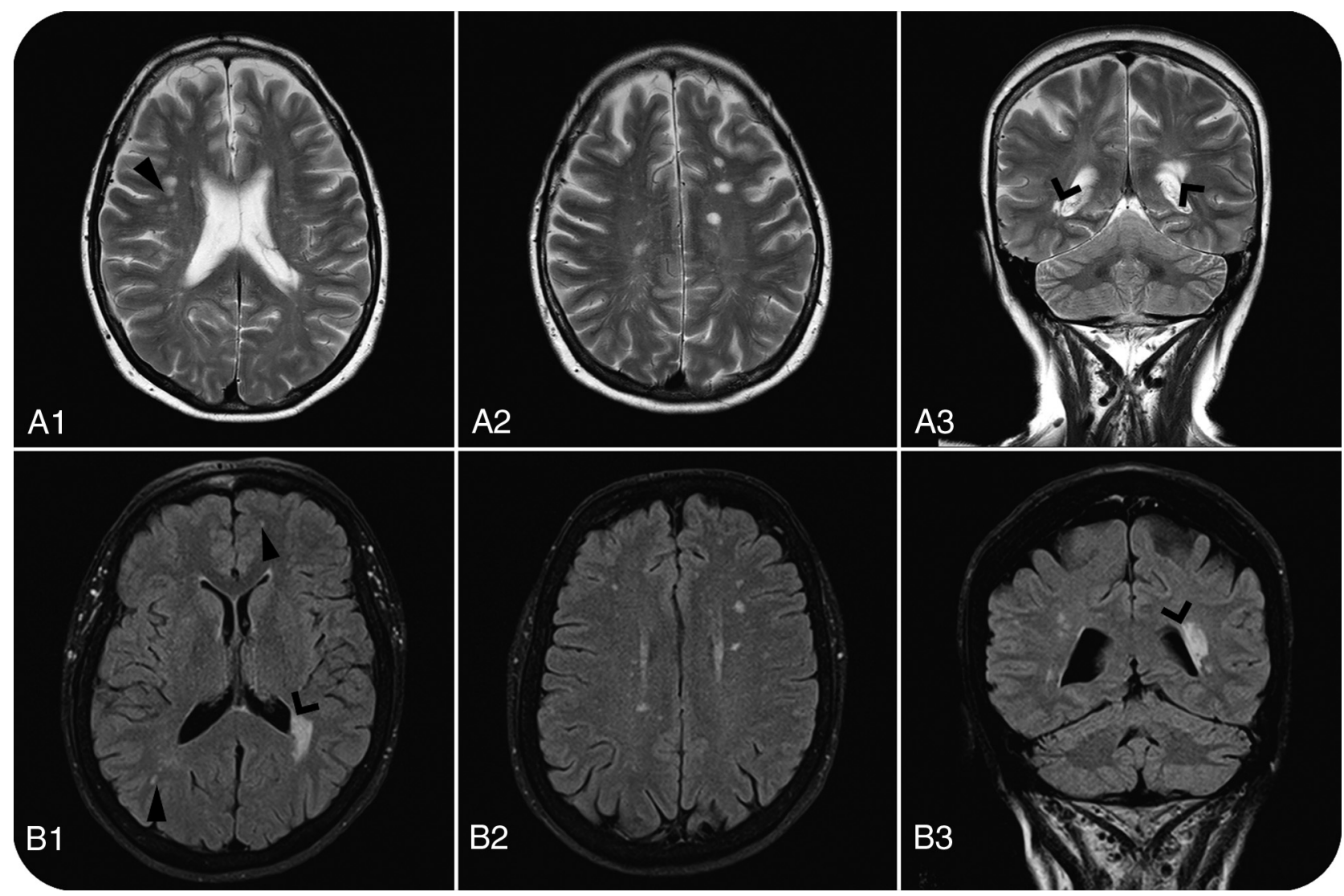

FIG 3. Brain MR imaging WM anomalies in 61-year-old (A1-A3: T2WI) and 66-year-old (BI-B3: FLAIR) women with RIS. Open arrows show periventricular lesions, and closed arrows show juxtacortical lesions, which, together with $>9$ lesions, made both patients fulfill the DIS-Barkhof and DIS-Swanton criteria. Columns 1 and 2 are axial sections; column 3 shows coronal sections.

no clinical indication existed. In those who had the analysis, 1 CSF sample was positive for oligoclonal bands.

After exclusion through the Okuda B-D and F criteria, 2 patients with RIS were identified fulfilling both classifications for DIS, while none of the patients fulfilling only the DIS-Swanton criteria remained. Thus, of the 1907 patients undergoing brain MR imaging in 2013, 2 patients fulfilled the criteria for RIS, equivalent to a cumulative incidence of $0.1 \%$ per year and a populationbased incidence of 0.8 cases of RIS per 100,000 inhabitants per year.

\section{Description of Individuals with RIS}

The first individual with RIS was an otherwise healthy 61-year-old woman referred for brain MR imaging due to headache and mild unspecific vertigo. In our blinded neuroradiologic re-evaluation, we found 3 periventricular lesions, 2 juxtacortical lesions, and $>9$ T2 lesions in total, thus fulfilling both DIS classifications. There were no contrast-enhancing lesions. CSF analysis showed immunoglobulin $\mathrm{G}$ oligoclonal bands but normal values of the immunoglobulin $\mathrm{G}$ index. There were no abnormal neurologic findings, and the patient did not recall having any earlier signs consistent with MS.

The second individual with RIS was a 66-year-old woman with hypothyroidism and asthma. She was referred for brain MR imaging because of suspected epilepsy with 2 self-reported episodes of generalized tonic-clonic seizures. The findings of electroencephalography were normal, and the suspicion of epilepsy was not confirmed. In the neuroradiologic assessment, 3 periventricular, 1 juxtacortical, and $>9 \mathrm{~T} 2$ lesions were found, fulfilling both DIS classifications. Findings on CSF analysis were normal, and there was no history of remitting neurologic symptoms or any abnormalities in the neurologic examination. The imaging findings of both patients with RIS are shown in Fig 3. Conditions of both patients had, before the study, been detected in the clinical context; they were referred to a neurologist and informed about the MR imaging findings. The patients did not fulfill the McDonald criteria for MS clinically, and neither of the 2 patients received disease-modifying therapy. None of the patients had any signs or symptoms consistent with MS 1 year after the MR imaging.

\section{DISCUSSION}

Of 1907 patients undergoing MR imaging, 2 patients were found to have RIS $(0.1 \%)$, regardless of using the Barkhof or Swanton classification for DIS. This finding equals an RIS incidence rate of 0.8 per 100,000 person-years. In comparison, the incidence rate of MS in Sweden is 10.2 per 100,000 person-years. ${ }^{23}$

Studies on the epidemiology of RIS are scarce. The few studies conducted have been hospital-based or studied the prevalence of RIS in selected cohorts such as in MS relatives. ${ }^{17,22,28}$ In a study of unexpected MR imaging findings suggestive of MS in a tertiary hospital in Pakistan, a frequency of $0.7 \%$ was reported in the age group 15-40 years. ${ }^{22}$ In a hospital-based study performed by us at a tertiary hospital in Stockholm, Sweden, we found an RIS fre- 
quency of $0.05 \%$ in all ages and $0.15 \%$ in the same age range as that in the study in Pakistan. ${ }^{17}$ Our studies do not overlap because they were performed with different sample years in different counties. The RIS frequency in the current study is in line with the reported hospital-based RIS frequency in our previous study. Meanwhile, the study from Pakistan showed a higher frequency of RIS, despite a lower incidence and prevalence of MS in the region. $^{23,24,30,31}$ Gabelic et $\mathrm{al}^{28}$ showed, in a smaller study in relatives of patients with MS, that $3 \%$ fulfilled the RIS criteria by using the DIS-Barkhof criteria, and 10\%, with the DIS-Swanton criteria. In healthy volunteers, they found RIS in $2.4 \%$ according to the DIS-Barkhof and $3.7 \%$ by using DIS-Swanton criteria. In the current study, $2.2 \%$ fulfilled the DIS-Swanton, and $1.0 \%$, the DISBarkhof criteria after neuroradiologic assessment (Okuda A and E), while no difference in RIS incidence $(0.1 \%)$ remained after exclusion according to clinical data (the Okuda B-D and F).

Possible explanations for the differences in RIS frequencies include methodologic differences (prospective/retrospective design, selection, and ages of study samples), regional differences in MR imaging practices, and availability and technical differences (field strengths, MR imaging sequences and orientation, section thickness, frequency of using contrast media). In our study, 2D FLAIR sequences were used and only approximately half of the MR imaging scans were obtained with gadolinium contrast media, which might influence the number of patients fulfilling DIS according to the Barkhof classification, in which the presence of enhancing lesions can substitute for 9 T2-hyperintense lesions. Increased awareness of MS signs and symptoms, both in the general population and among physicians, in regions with a high incidence of MS is also likely to influence the rate of unexpected MS-like findings. An alternative interpretation could be that the RIS frequency may be higher in regions with lower MS incidence if differences in the genetic or environmental background reduce the risk of converting to manifest MS. If that interpretation is the case, studying the difference between patients with RIS and those with MS is of major importance, to understand the mechanisms that may constrain the development of MS.

The individuals with RIS in our study had a surprisingly older age, considering that the incidence for women with MS in Sweden peaks at 30 years of age. ${ }^{23}$ These results suggest that RIS may reflect a more benign entity, but longitudinal data on the natural history of RIS are needed to better understand its prognosis. ${ }^{4,5,11,18}$ Whether individuals with RIS should receive MS treatment is debated, ${ }^{32,33}$ but a clinical treatment trial is already planned. ${ }^{34}$ Due to the low incidence of RIS, conducting studies on patients with RIS may necessitate large nation-based cohorts or multicenter studies. Our results may be of importance when designing future treatment trials and longitudinal studies on the natural history of RIS.

The main strength of our study is the population-based perspective, being the first study of RIS with a known population size and an involvement of all MR imaging centers in the area. The design reduces the risk of selection bias, which has been considered the main limitation of previous hospital-based studies. The comprehensive inclusion of all performed MRIs in a region, such as in this study, can yield the incidence rate of RIS, because RIS is defined as an incidental MR imaging finding. Our results of RIS incidence are representative of the clinical entity RIS, which causes clinical dilemmas in terms of management, but does not reflect an estimation of the prevalence of RIS in the general population, in which most had not undergone a brain MR imaging. The selection bias of the imaged individuals most likely yields a higher RIS incidence among the imaged than the nonimaged population. If the imaged population were representative of the nonimaged one, the frequency of RIS would be 105 cases per 100,000 person-years (based on the 2 cases of RIS per 1907 persons), which would be $>10$ times larger than the MS incidence (10.2 cases per 100,000 person-years).

An additional strength is the blinded radiologic readings performed by a senior radiologist and a senior neuroradiologist. The high accessibility of the clinical patient charts and clinical information was essential for the assessment regarding Okuda B-D and $\mathrm{F}$ and for not overestimating the RIS incidence.

A limitation is the use of different MR imaging scanners and MR imaging protocols, which may have different sensitivities for WM lesions. This variability is, however, a consequence of the natural variation of equipment used at different centers and increases the generalizability of the results because scanner variations are common in clinical practice. The main limitation of the study is its sample size, because the relative sparseness of RIS renders the incidence estimate to some degree uncertain. Future epidemiologic RIS studies would therefore benefit from longer study periods or a larger study population.

\section{CONCLUSIONS}

Our study shows that RIS is relatively uncommon in a high-incidence region for MS. Future studies on RIS may therefore benefit from a collaborative approach to ensure adequate numbers of participants. There was no difference in RIS incidence when applying the newer Swanton classification for DIS, suggesting that it may be feasible to adapt the Okuda criteria to harmonize with the 2010 MacDonald MS criteria, without affecting the incidence of RIS.

\section{ACKNOWLEDGMENTS}

We thank the Department of Radiology at Västmanland Hospital and Medicinsk Röntgen Eriksborg for participating in the study, and we especially thank Per Hammar and Arne Thorén, the directors of the respective clinics, for facilitating this study.

Disclosures: Yngve Forslin, Ayad Antwan Jumah—RELATED: Grant: This research was funded by Stockholm County Council and Karolinska Institutet through the regional agreement on medical training and clinical research (ALF grant 20120213). Sten Fredrikson—UNRELATED: Board Membership: Teva, Genzyme; Payment for Lectures (including service on Speakers Bureaus): Allergan, Bayer, Biogen Idec, Genzyme, Merck Serono, Novartis, Sanofi, Teva; Payment for Development of Educational Presentations: Teva; Other: received honoraria for lectures, educational activities, or consultancy from Allergan, Bayer, Biogen Idec, Genzyme, Merck Serono, Novartis, Sanofi, and Teva.

\section{REFERENCES}

1. OECDiLibrary. Magnetic resonance imaging (MRI) exams, total per 1000 population. www.oecd-ilibrary.org/content/table/mri-examtotal-table-2014-1-en. Accessed June 18, 2014

2. Morris Z, Whiteley WN, Longstreth WT, et al. Incidental findings on brain magnetic resonance imaging: systematic review and metaanalysis. BMJ 2009;339:b3016 CrossRef Medline 
3. Okuda DT, Mowry EM, Beheshtian A, et al. Incidental MRI anomalies suggestive of multiple sclerosis: the radiologically isolated syndrome. Neurology 2009;72:800-05 CrossRef Medline

4. Barkhof F, Filippi M, Miller DH, et al. Comparison of MRI criteria at first presentation to predict conversion to clinically definite multiple sclerosis. Brain 1997;120(pt 11):2059-69 CrossRef Medline

5. Granberg T, Martola J, Kristoffersen-Wiberg M, et al. Radiologically isolated syndrome: incidental magnetic resonance imaging findings suggestive of multiple sclerosis-a systematic review. Mult Scler 2013;19:271-80 CrossRef Medline

6. Lebrun C, Blanc F, Brassat D, et al; CFSEP. Cognitive function in radiologically isolated syndrome. Mult Scler 2010;16:919-25 CrossRef Medline

7. De Stefano N, Stromillo ML, Rossi F, et al. Improving the characterization of radiologically isolated syndrome suggestive of multiple sclerosis. PLoS One 2011;6:e19452 CrossRef Medline

8. Giorgio A, Stromillo ML, Rossi F, et al. Cortical lesions in radiologically isolated syndrome. Neurology 2011;77:1896-99 CrossRef Medline

9. Amato MP, Hakiki B, Goretti B, et al; Italian RIS/MS Study Group. Association of MRI metrics and cognitive impairment in radiologically isolated syndromes. Neurology 2012;78:309-14 CrossRef Medline

10. Stromillo ML, Giorgio A, Rossi F, et al. Brain metabolic changes suggestive of axonal damage in radiologically isolated syndrome. Neurology 2013;80:2090-94 CrossRef Medline

11. Lebrun C, Bensa C, Debouverie M, et al; CFSEP. Unexpected multiple sclerosis: follow-up of 30 patients with magnetic resonance imaging and clinical conversion profile. J Neurol Neurosurg Psychiatry 2008;79:195-98 CrossRef Medline

12. Derwenskus Joy, Cohen B. Clinically silent multiple sclerosis: description of a patient cohort without symptoms typical of MS but abnormal brain magnetic resonance imaging. Multiple Sclerosis (Houndmills, Basingstoke, England) 2007;3:1226-27

13. Lebrun C, Bensa C, Debouverie M, et al; Club Francophone de la Sclérose en Plaques. Association between clinical conversion to multiple sclerosis in radiologically isolated syndrome and magnetic resonance imaging, cerebrospinal fluid, and visual evoked potential: follow-up of 70 patients. Arch Neurol 2009;66:841-46 CrossRef Medline

14. Siva A, Saip S, Altintas A, et al. Multiple sclerosis risk in radiologically uncovered asymptomatic possible inflammatory-demyelinating disease. Mult Scler 2009;15:918-27 CrossRef Medline

15. Okuda DT, Mowry EM, Cree BA, et al. Asymptomatic spinal cord lesions predict disease progression in radiologically isolated syndrome. Neurology 2011;76:686-92 CrossRef Medline

16. Maia AC Jr, Rocha AJ, Barros BR, et al. Incidental demyelinating inflammatory lesions in asymptomatic patients: a Brazilian cohort with radiologically isolated syndrome and a critical review of current literature. Arq Neuropsiquiatr 2012;70:5-11 CrossRef Medline

17. Granberg T, Martola J, Aspelin P, et al. Radiologically isolated syndrome: an uncommon finding at a university clinic in a highprevalence region for multiple sclerosis. BMJ Open 2013;3:e003531 CrossRef Medline
18. Okuda DT, Siva A, Kantarci O, et al; Radiologically Isolated Syndrome Consortium (RISC), Club Francophone de la Sclérose en Plaques (CFSEP). Radiologically isolated syndrome: 5-year risk for an initial clinical event. PLoS One 2014;9:e90509 CrossRef Medline

19. Gilbert JJ, Sadler M. Unsuspected multiple sclerosis. Arch Neurol 1983;40:533-36 CrossRef Medline

20. Engell T. A clinical patho-anatomical study of clinically silent multiple sclerosis. Acta Neurol Scand 1989;79:428 -30 CrossRef Medline

21. Johannsen LG, Stenager E, Jensen K. Clinically unexpected multiple sclerosis in patients with mental disorders: a series of 7301 psychiatric autopsies. Acta Neurol Belg 1996;96:62-65 Medline

22. Wasay M, Rizvi F, Azeemuddin M, et al. Incidental MRI lesions suggestive of multiple sclerosis in asymptomatic patients in Karachi, Pakistan. J Neurol Neurosurg Psychiatry 2011;82:83-85 CrossRef Medline

23. Ahlgren C, Odén A, Lycke J. High nationwide incidence of multiple sclerosis in Sweden. PLoS One 2014;9:e108599 CrossRef Medline

24. Ahlgren C, Odén A, Lycke J. High nationwide prevalence of multiple sclerosis in Sweden. Mult Scler 2011;17:901-08 CrossRef Medline

25. Statistics Sweden. Population in the country, counties and municipalities by sex and age December 31, 2014. http://www.scb.se/en_/Findingstatistics/Statistics-by-subject-area/Population/Population-composition/ Population-statistics/Aktuell-Pong/25795/Yearly-statistics-MunicipalitiesCounties-and-the-whole-country/370301/. Accessed January 27, 2015

26. Swanton JK, Rovira A, Tintore M, et al. MRI criteria for multiple sclerosis in patients presenting with clinically isolated syndromes: a multicentre retrospective study. Lancet Neurol 2007;6:677-86 CrossRef Medline

27. Polman $\mathrm{CH}$, Reingold SC, Banwell B, et al. Diagnostic criteria for multiple sclerosis: 2010 revisions to the McDonald criteria. Ann Neurol 2011;69:292-302 CrossRef Medline

28. Gabelic T, Ramasamy DP, Weinstock-Guttman B, et al. Prevalence of radiologically isolated syndrome and white matter signal abnormalities in healthy relatives of patients with multiple sclerosis. AJNR Am J Neuroradiol 2014;35:106-12 CrossRef Medline

29. Liu S, Kullnat J, Bourdette D, et al. Prevalence of brain magnetic resonance imaging meeting Barkhof and McDonald criteria for dissemination in space among headache patients. Mult Scler 2013;19: 1101-05 CrossRef Medline

30. Wasay M, Khatri IA, Khealani B, et al. MS in Asian countries. Int MS J 2006;13:58-65 Medline

31. Wasay M, Ali S, Khatri IA, et al. Multiple sclerosis in Pakistan. Mult Scler 2007;13:668-69 CrossRef Medline

32. Brassat D, Lebrun-Frenay C; Club Francophone de la SEP. Treat patients with radiologically isolated syndrome when the MRI brain scan shows dissemination in time: yes. Mult Scler 2012;18:1531-32 CrossRef Medline

33. Bourdette $\mathrm{D}$, Yadav V. Treat patients with radiologically isolated syndrome when the MRI brain scan shows dissemination in time no. Mult Scler 2012;18:1529-30 CrossRef Medline

34. Okuda D, Frenay CL, Siva A, et al. Multi-center, randomized, double-blinded assessment of dimethyl fumarate in extending the time to a first attack in radiologically isolated syndrome (RIS) (ARISE Trial) (P7.207). Neurology 2015;84(suppl):P7.207 